\title{
Belantamab Mafodotin for the Treatment of Multiple Myeloma: An Overview of the Clinical Efficacy and Safety
}

\author{
Massimo Offidani ${ }^{\prime}$ \\ Laura Corvatta ${ }^{2}$ \\ Sonia Morè \\ Attilio Olivieri ${ }^{1}$
}

'Clinica di Ematologia Azienda

Ospedaliero-Universitaria, Ospedali

Riuniti di Ancona, Ancona, Italy; ${ }^{2}$ U.O.C.

Medicina, Ospedale Profili, Fabriano, Italy

\begin{abstract}
Despite the introduction of immunomodulatory drugs (IMiDs), proteasome inhibitors (PIs), and, more recently, monoclonal antibodies (mAbs), in the chemotherapy regimens for newly diagnosed (NDMM) and relapsed/refractory MM (RRMM), the occurrence of drug resistance remains a challenge in MM patients. This is mainly in the advanced stage of the disease when treatments are limited, and the prognosis is abysmal. Nevertheless, novel molecules and therapeutic approaches are rapidly moving through the several phases of drug development and could address the need for new treatment options. The recent innovative B-cell maturation antigen (BCMA) targeted immunotherapies, such as belantamab mafodotin, the first-in-class monoclonal antibody-drug conjugate (ADC), induce an effective and durable response in triple-class refractory disease and to be approved in MM. In contrast with the other BCMA-targeted therapies as CAR T cells with a complex manufacturing process, and bispecific antibodies, both requiring inpatient hospitalization to monitor the occurrence of severe adverse events, belantamab mafodotin is an "off-the-shelf" drug that can be administered in an outpatient setting. Many belantamab mafodotin-based combinations are under evaluation in Phase I, II, and III clinical trials either late or in early RRMM patients. Ocular toxicity represents a peculiar side effect of belantamab mafodotin. This toxicity is generally manageable with adequate dose reductions or delays since most patients who developed keratopathy recovered on treatment and discontinued ADC are rare. Here, we described the most recent clinical data of belantamab mafodotin and discussed the possible leading role of this intriguing agent in the near future of MM treatment.
\end{abstract}

Keywords: multiple myeloma, relapsed, refractory, belantamab mafodotin, antibody-drug conjugate

\section{Introduction}

Multiple Myeloma (MM), the second most frequent hematological neoplasm, ${ }^{1}$ is characterized by aberrant proliferation of plasma cells, which commonly produce a large number of monoclonal immunoglobulins leading to organ damage, such as osteolytic bone lesions and renal impairment, as well as bone marrow failure symptoms like anemia or recurrent infection due to immune-paresis. ${ }^{2}$ Although MM remains incurable, it is a biologically very heterogeneous disease with median survival ranging from 2 to 10 years.,

The therapeutic landscape of MM has outstandingly changed in the last 20 years with the introduction of proteasome inhibitors (PIs: bortezomib, carfilzomib, ixazomib), immunomodulatory agents (IMiDs: thalidomide, lenalidomide,
Correspondence: Massimo Offidan Clinica di Ematologia Azienda Ospedaliero-Universitaria, Ospedali Riuniti di Ancona, Via Conca, 7I, Ancona, 60020 , Italy

Tel +3907I5964735

Email massimo.offidani@ospedaliriuniti. marche.it 
pomalidomide), and, more recently, monoclonal antibodies (mAbs: elotuzumab, daratumumab, isatuximab), which has led to an unprecedented improvement of the outcome either in patients eligible (TE) or not-eligible for transplantation (NTE). Currently, therapeutic approaches including triplet induction combinations, high-dose therapy (HDT) followed by autologous transplantation (ASCT), and consolidation-maintenance, results in a 10years survival probability of $60 \%$ in TE patients. ${ }^{5}$ In elderly patients, triplet or doublet combinations with PIs or IMiDs allowed achieving a median survival of 5 years. ${ }^{6}$ Perhaps the addition of daratumumab, mAb targeting CD38 molecule, to regimens used in the upfront setting could further improve survival both in TE and NTE patients. However, the course of MM is characterized by periods in which the disease is in remission and by phases of relapse until MM becomes resistant to therapies. At this point, $\mathrm{MM}$ is complicated to treat since the several drug combinations used in earlier lines of treatment further reduce the availability of subsequent effective treatments. $^{7}$ Patients who become refractory to PIs, IMiDs, and monoclonal antibodies have an inferior outcome with a median OS of 5.6 months $^{8}$, so there is a significant unmet need for effective, innovative therapies to be offered to these patients. Among novel immunotherapies, belantamab mafodotin (Belamaf) is the first antibody-drug conjugate targeting B-cell maturation antigen (BCMA) to be approved for the treatment of multiple myeloma. This review will focus on Belamaf, describing its mechanism of action, pharmacokinetics, and available clinical data on their safety and efficacy.

\section{Methods}

To perform this review, we searched the database of PubMed using the terms "multiple myeloma", "relapsed", "refractory", "B-cell maturation antigen", "antibody-drug conjugate", "belantamab mafodotin". Available data included in abstracts presented at the ASH, ASCO, EHA, and other International Meetings were also used. Moreover, the clinicaltrials.gov database was searched using "multiple myeloma" and "belantamab mafodotin".

\section{Results}

\section{Emerging Therapies for RRMM}

After the therapeutic success of using mAbs to treat other hematologic malignancies, ${ }^{9,10}$ this approach became available for MM.
Elotuzumab, a humanized IgG1 mAb binding to SLAMF7 (signaling lymphocytic activation molecule 7) expressed both on MM and NK cells, is the first one to be explored in the treatment of $\mathrm{MM}^{11}$ and to demonstrate, in combination with lenalidomide and dexamethasone (Elo$\mathrm{Rd}$ ), a significant OS benefit in relapsed/refractory MM (RRMM) compared to lenalidomide plus dexamethasone (Rd) in Phase III ELOQUENT-2 trial. ${ }^{12}$ Triplets Elo-Rd and Elo-Pd (elotuzumab, pomalidomide, and dexamethasone), assessed in phase III ELOQUENT-3 study $^{13}$ have been approved for the treatment of RRMM.

Daratumumab is the first fully human $\mathrm{IgG} 1 \kappa$ targeting CD38 eliciting cell death through complement-dependent cytotoxicity (CDC), antibody-dependent cell-mediated cytotoxicity (ADCC), antibody-dependent cellular phagocytosis (ADCP), induction of apoptosis, and modulation of CD38 enzyme activities. ${ }^{14}$ After GEN50 $1{ }^{15}$ and SIRIUS ${ }^{16}$ studies demonstrated the efficacy of daratumumab monotherapy; it has been approved in combinations with lenalidomide and dexamethasone (DRd) or Bortezomib and dexamethasone (DVd) for treatment of RRMM who have received at least one prior line of therapy, based on the results of phase III POLLUX ${ }^{17}$ and CASTOR ${ }^{18}$ trials. More recently, daratumumab has been evaluated in combination with pomalidomide and dexamethasone (DPd) vs Pd or combination with Carfilzomib and dexamethasone $(\mathrm{DKd})$ vs $\mathrm{Kd}$ in phase III APOLLO ${ }^{19}$ and $\mathrm{CANDOR}^{20}$ trials. Both studies met their primary endpoint of improved PFS, and these combinations are starting to become the new standard in RRMM patients. However, with daratumumab combination regimens now increasingly utilized in the front-line setting based on phase III CASSIOPEIA, ${ }^{21}$ $\mathrm{ALCYONE}^{22}$, and MAIA ${ }^{23}$ trials, the role of daratumumab-containing therapies in the relapse setting is uncertain, in the absence of clear evidence that rechallenging with daratumumab can overcome prior daratumumab refractoriness.

Another mAb targeting CD38 is isatuximab, a chimeric IgG1 $\mathrm{mAb}$ targeting a specific epitope on CD38. This mechanism of action is different from that of daratumumab since it induces direct apoptosis in MM cells, inhibits CD38 enzymatic activity more effectively than daratumumab, and is less able to induce CDC. Moreover, isatuximab exerts an immunomodulatory effect since it inhibits the suppressive function of Tregs by reducing their number, decreasing immune inhibitory cytokine production, including IL-10, and blocking their trafficking. All these activities improved NK- and T-cell-mediated antitumor 
immune responses. ${ }^{24}$ Isatuximab in combination with pomalidomide and dexamethasone (Isa-Pd) can be used in RRMM patients who have received at least two prior lines of therapy after approval based on results of phase III ICARIA trial. $^{25}$

However, as with other agents, resistance to daratumumab is a possible event leading to a poor outcome, as showed in the retrospective MAMMOTH study $^{8}$ including 275 patients among whom those penta-refractory (refractory to $1 \mathrm{CD} 38 \mathrm{mAb}+2 \mathrm{PIs}+2 \mathrm{IMiDs}$ ) had a median OS of 5.6 months. Hence, there is a need for developing and introducing novel therapies in clinical practice. Among more advanced emerging treatments, selinexor, melfuflen, cerebron E3 ligase modulators (CELMoDs), and venetoclax were found very promising. Selinexor is the first-in-class oral selective inhibitor Nuclear Export (SINE) compound targeting XPO-1 (exporting-1) to be explored in $\mathrm{MM}^{26}$ Recently, it has been approved in combination with Bortezomib and dexamethasone (SVd) for treatment of RRMM patients who have received at least one prior therapy, based on results of phase III BOSTON trial. ${ }^{27}$ In patients treated with one to three previous lines of treatment, SVd allowed a median PFS of 13.9 months vs 9.5 months in patients treated with $\mathrm{Vd}$. Besides grade 3-4, thrombocytopenia and neutropenia occurring in $39 \%$ and $6 \%$ of patients, respectively, about $25 \%$ of patients experienced gastrointestinal toxicity. In 2019 selinexor had received approval in combination with dexamethasone in advanced disease. ${ }^{28}$ Melfuflen (melfuflen-flufenamide) is the first-in-class peptide-drug conjugate targeting aminopeptidases and releasing alkylating agents into tumor cells ${ }^{29}$ which demonstrated in combination with dexamethasone efficacy in triple-class refractory MM patients in the Phase II HORIZON, showing in RRMM patients with a median of 5 prior lines of therapy a median PFS of 4.2 months. ${ }^{30}$ Combined with dexamethasone, it has been recently approved by FDA in patients who have received at least four previous lines of therapy. CELMoDs starting from iberdomide represent the nextgeneration IMiDs and exert more potent immunomodulation than lenalidomide and pomalidomide. ${ }^{31}$ Venetoclax, an oral BCL-2 targeted therapy, approved for CLL and AML, demonstrated remarkable efficacy in RRMM harboring $t$ $(11 ; 14)$ or with high BCL-2 expression. ${ }^{32}$

Novel immunotherapies, including bispecific antibodies, antibody-drug conjugates, and CAR T cells, human genetically modified $\mathrm{T}$ cells, are the new frontiers of MM therapy and great hope in more advanced and refractory diseases in which these treatments can induce high-quality and durable responses. $^{33,34}$ In the recently published KarrMMa study, ${ }^{35}$ idecabtagene vicleucel at the dose of $450 \times 10^{6}$ CAR T cells induced responses in $81 \%$ of patients who had received a median of 6 prior lines of therapies and median PFS was 12.1 months. As regard BCMA targeting bispecific antibodies, preliminary results from teclistamab given subcutaneously showed a promising ORR of $73 \%$ in patients with a median of 5 previous lines of therapy, being ORR $75 \%$ in penta-refractory patients. ${ }^{36}$

The availability of all these therapeutic options in the near future will make the choice of treatment in RRMM a very complex process.

\section{Chemistry and Mechanisms of Action of} Belantamab Mafodotin

As mentioned above, naked mAbs eliminate target MM cells by activating host defense mechanisms regulated by Fc $\gamma$ receptor-expressing effector cells. ${ }^{37}$ However, in MM patients, immune dysregulation involving $\mathrm{B}$ cells and T cells, dendritic cells (DC), and natural killer cells (NK) has been demonstrated. ${ }^{38}$ Daratumumab was found to induce expansion of T-cells and increase T-cell clonality. Still, in patients who become resistant to this $\mathrm{mAb}$, an increase of exhausted $\mathrm{T}$ cells with upregulation of the checkpoint inhibitors lymphocyte-activation gene 3 (LAG3) and T cell immunoreceptor with Ig and ITIM domains (TIGIT) was observed. ${ }^{39}$ Moreover, NK cell depletion has been documented following daratumumab administration and may reduce the efficacy of this $\mathrm{mAb}^{40}$ An evolution of naked mAbs is represented by antibody-drug conjugates (ADC), a payload delivery system is consisting of a monoclonal antibody directed against a tumor-specific antigen and a cytotoxic agent inducing cell death (payload) covalently conjugated through chemical linkers ${ }^{41}$ (Figure 1). An ideal antigen for effective immunotherapy with ADC should be a highly expressed molecule on the membrane of MM plasma cells, and BCMA, a member of the TNF receptor superfamily 17 , represents an excellent candidate since it has been found on the surface of late-stage of normal B lymphocytes and malignant plasma cells. ${ }^{42}$ For BCMA, two ligands have been identified: a proliferationinducing ligand (APRIL) and B-cell activating factor (BAFF). ${ }^{43}$ Activation of BCMA enhances expression of genes critical for the survival of plasma cell growth, adhesion, osteoclast activation, angiogenesis, metastasis, and immunosuppression. ${ }^{44}$ Soluble BCMA (sBCMA) derives 


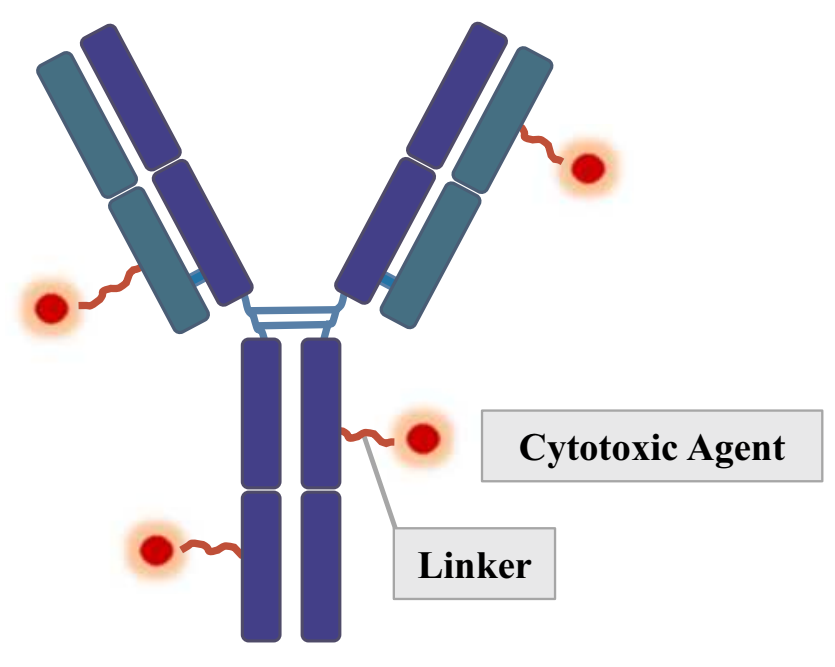

Monoclonal Antibody

Figure I Structure of an antibody-drug conjugate.

from the ectodomain shedding of the membrane receptor by gamma-secretase activity. The levels of sBCMA have prognostic value ${ }^{45}$ correlate with the phase of MM's natural history, increasing through the progression from MGUS to smoldering myeloma and then to active $\mathrm{MM} ;{ }^{46}$ they can also be helpful to monitor clinical response to ongoing therapy. ${ }^{45,47}$

The choice of the antibody is crucial and, based on experience with naked mAbs as elotuzumab and daratumumab, the most commonly used antibody is a fully human or humanized antibody immunoglobulin $\mathrm{G}$ subtype (IgG) for long-circulating half-life in the human bloodstream (up to three weeks) and minimal immunogenicity. Probably, the most complex aspect of ADC development is the chemistry of the linker requiring several requirements: the linker has to be strong enough to withstand degradation in the plasma since instability of linker causes premature liberation of toxic payload with damage to non-target healthy cells; in the same time, it has to be vulnerable enough for degradation and release of the payload in the endosome/lysosome once the ADC is internalized into the target tumor cell. ${ }^{41,48}$ Linkers can be categorized into two classes according to the payload release mechanism: cleavable linkers are designed to respond to a specific environmental condition $(\mathrm{pH}$, lysosomal protease, or glutathione) which assesses the linkers to enable chemical or biochemical reactions as hydrolyzation or proteolysis. Non-cleavable linkers rely on the monoclonal antibody degradation by cytosolic and lysosomal proteases after ADC internalization with the liberation of payload molecule linked with an amino acid residue of degraded antibody. ${ }^{41}$ It is also necessary to pay attention to the choice of the killing molecule that requires several requirements as substantial cell toxicity, possessing the appropriate modified site from where the conjugate releases the original drug in the tumor cell, and a definite mechanism of action. ${ }^{49}$ The commonly used payloads are microtubule inhibitor agents as maytansinoids and auristatin (including monomethyl auristatin $\mathrm{E}$ and $\mathrm{F}$ ), able to bind to tubulin, causing G2/M arrest and apoptosis ${ }^{50}$ and DNAmodifying drugs as calicheamicin, inducing cell death by DNA double-strand breaking. ${ }^{51}$ In the next future, payloads will probably be less potent but more targeted against a specific tumor cell. In Table 1, we summarized the main antibody-drug conjugates in development.

Belantamab mafodotin (Belamaf, GSK2587916) is a first-in-class humanised IgG1 ADC developed to treat MM. This afucosylated Fc-engineered ADC includes an antibody targeting BCMA covalently linked via a cysteine linker to the microtubule inhibitor monomethyl auristatin F (MMAF). Therefore, the most important characteristics of Belamaf structure are the highly potent cytotoxic agent; the afucosylation significantly increasing the binding affinity of the Fc domain to the Fc $\gamma \mathrm{R}$ (Fc $\gamma$ RIIIa) expressed on effector cells; the uncleavable maleimidocaproyl (mc) cysteine linker region, which makes the ADC stable in circulation. ${ }^{52}$ Several are the mechanism of action of Belamaf, and the main one is binding to BCMA on tumor plasma cell, internalization, and release of MMAF, which binds to tubulin and inhibits its polymerization resulting in G2/M arrest and caspase 3 dependent apoptosis (Figure 2). ${ }^{53}$ Due to afucosylation of antibody, Belamaf

Table I Main Antibody Drug Conjugates Targeting BCMA

\begin{tabular}{|l|c|c|c|}
\hline Agent & Target & Type & Phase \\
\hline AMG224 & BCMA & Mertansine ADC & $\mathrm{I}$ \\
\hline MEDI2228 & BCMA & Pyrrolobenzodiazepine ADC & $\mathrm{I}$ \\
\hline $\begin{array}{l}\text { CC- } \\
99,712\end{array}$ & BCMA & Undisclosed ADC & $\mathrm{I}$ \\
\hline HDP-10I & BCMA & Amanitin ADC & Preclinical \\
\hline STRO-00I & CD74 & Maytansinoid ADC & $\mathrm{I}$ \\
\hline FOR46 & CD46 & monomethyl auristatin-F ADC & $\mathrm{I}$ \\
\hline ABBV-838 & SLAMF7 & monomethyl auristatin-E ADC & $\mathrm{I} / \mathrm{lb}$ \\
\hline
\end{tabular}




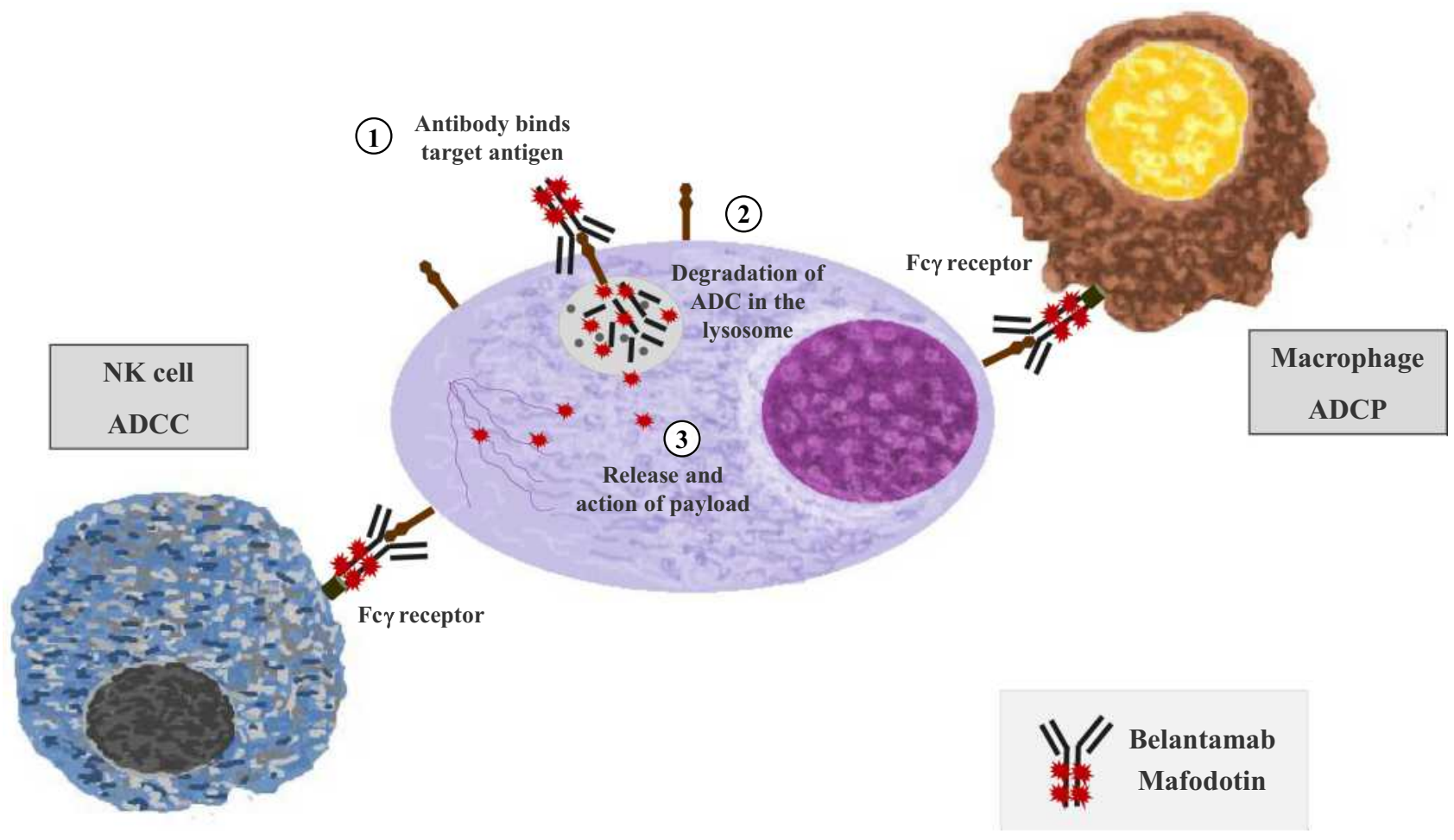

Figure 2 Mechanisms of action of belantamab mafodotin.

significantly improves the potency and efficacy of ADCC activity against $\mathrm{MM}$ cells. Importantly, in vitro studies showed that ADCC was enhanced by the addition of lenalidomide, providing the basis for a possible combination of Belamaf with IMiDs.

Moreover, Belamaf exerts anti-myeloma activity inducing ADCP. A dose of $4 \mathrm{mg} / \mathrm{kg}$ was found in mouse xenograft models to induce a complete tumor eradication for the entire 60-day study. ${ }^{53}$ More recently, in vitro and in vivo studies demonstrated that durable antitumor activity of Belamaf is characterized by $\mathrm{T}, \mathrm{NK}$, and dendritic cell infiltration and increased markers of immunogenic cell death (ICD) and is abrogated upon depletion of CD8+ $\mathrm{T}$ cells. ${ }^{54}$ Therefore, these mechanisms represent a rationale for combination with various immunomodulatory therapies.

\section{Clinical Studies DREAMM-I Study}

Phase I DREAMM-1 ${ }^{55}$ was the first-in-human study to explore Belamaf as monotherapy in RRMM. This international study comprised a Part 1, a dose-escalation phase assessing the safety and tolerability of Belamaf and recommended phase II dose (RP2D) and a Part 2, doseexpansion phase, evaluating safety and tolerability pharmacokinetics, and clinical activity. In part 1, patients received Belamaf at doses ranging from $0.03 \mathrm{mg} / \mathrm{kg}$ to $4.6 \mathrm{mg} / \mathrm{kg}$, administered in $1 \mathrm{~h}$ intravenous infusion every three weeks until progression, unacceptable toxicity, or completion of 16 doses of treatment. To evaluate the incidence and severity of infusion-related reactions (IRRs), prophylaxis was not permitted for the first infusion but only for subsequent ones. From 2014 to 2017, 73 patients were enrolled, 38 in Part 1 and 35 in Part 2. Among patients included in the dose-escalation phase, $76 \%$ had received at least five prior lines of therapy and the median time from diagnosis was 5.3 years. The most common grade 3-4 adverse events were thrombocytopenia (34\%) and anemia (16\%). However, $53 \%$ of patients receiving Belamaf developed ocular toxicity, which is a well-known side effect with other MMAF-linked antibody-drug ${ }^{56}$ and will be described in more detail in a chapter below. No maximal tolerated dose was established, so $3.4 \mathrm{mg} / \mathrm{kg}$ was the RP2D to treat patients in Part 2 of the study, chosen based on pharmacokinetics, activity, and safety data. Regarding characteristics of 35 patients enrolled in Part 2, ${ }^{57} 40 \%$ had received more than five prior lines of therapy, $97 \%$ were refractory to PIs, $94 \%$ to IMiDs, $40 \%$ to daratumumab. The most common grade 3-4 adverse events were thrombocytopenia (35\%), anemia 
(17/\%), pneumonia (6\%), and IRRs $(6 \%)$. Corneal events were seen in $69 \%$ of patients, most commonly blurred vision (51\%), dry eye (37\%), and photophobia (29\%), whose median duration was 35 days. Regarding efficacy data, $60 \%$ of patients achieved a PR or better, and $54 \%$ achieved at least VGPR. In patients without and with prior daratumumab exposure, ORR was $71.4 \%$ and $42.9 \%$, respectively. In contrast, ORR was $38.5 \%$ in triplerefractory (daratumumab $+1 \mathrm{PI}+1 \mathrm{IMiD}$ ), $70 \%$ in patients who had received $\leq 3$ prior therapies and $50 \%$ in $>5$ prior therapies. The median time to first response was 1.2 months, and responses deepened over time. After over a year of additional follow-up from the previous interim analyses performed with a median follow-up of 12.5 months, the median duration of response was 14.3 months and median PFS 12 months. In patients with prior daratumumab treatment and refractory disease to PIs and IMiDs, the median PFS was 6.2 months. In patients without daratumumab treatment, median PFS was 15.7 months. ${ }^{57}$

\section{DREAMM-2 Study}

The most important study leading to Belamaf approval for RRMM treatment is the international, open-label, twoarm, phase II DREAMM-2 study $^{58}$ which explored the safety and efficacy of two doses of belantamab mafodotin in RRMM patients who were refractory to an IMiDs (lenalidomide or pomalidomide), a PI (Bortezomib, Carfilzomib or ixazomib) and refractory or intolerant to an anti-CD38 mAb. Dosing regimens explored were Belamaf $2.5 \mathrm{mg} / \mathrm{kg}$ or $3.4 \mathrm{mg} / \mathrm{kg}$, selected based on results of the DREAMM-1 study. The trial's primary endpoint was ORR, defined as the percentage of patients achieving at least PR as per IMWG 2016 criteria. $^{59}$ In contrast, secondary outcomes were duration of response (DoR), time to response, PFS, OS, and safety, including keratopathy. One hundred ninety-six patients were randomly allocated to receive Belamaf $2.5 \mathrm{mg} / \mathrm{kg} \quad(\mathrm{n}=97)$ and $3.4 \mathrm{mg} / \mathrm{kg}(\mathrm{n}=99)$ intravenously over $30 \mathrm{~min}$, every three weeks until disease progression or unacceptable toxicity. Patients had received a median of 7 (range 3-21) and 6 (range 3-21) prior lines of therapy in the $2.5 \mathrm{mg} / \mathrm{kg}$ and $3.4 \mathrm{mg} / \mathrm{kg}$ cohort, respectively. As per inclusion criteria, all patients were refractory to an IMiD, to a PI and $100 \%$ and $92 \%$ of patients were refractory to previous therapies including daratumumab in the $2.5 \mathrm{mg} / \mathrm{kg}$ and $3.4 \mathrm{mg} / \mathrm{kg}$ cohort, respectively, with $34 \%$ and $37 \%$ of patients receiving daratumumab as the last prior therapy. Patients with ISS stage III, extramedullary disease, and high-risk cytogenetics were well represented in both groups. At a median follow-up of 13 months, ORR was $31 \%$ in the $2.5 \mathrm{mg} / \mathrm{kg}$ cohort and $34 \%$ in the $3.4 \mathrm{mg} / \mathrm{kg}$ cohort. Belamaf monotherapy was found to induce deep responses since $19 \%$ and $23 \%$ (58\% and $66 \%$ of patients achieving at least PR) in the 2.5 and 3.4 groups, respectively, achieved at least VGPR. The median DoR and PFS were 11 months and 2.8 months in the $2.5 \mathrm{mg} / \mathrm{kg}$ group vs 6.2 months and 3.9 months in the $3.4 \mathrm{mg} / \mathrm{kg}$ group. Median OS was 13.7 months in the $2.5 \mathrm{mg} / \mathrm{kg}$ cohort and 13.8 months in the $3.4 \mathrm{mg} / \mathrm{kg}$ cohort with the estimated 1-year survival of approximately $57 \%$ in both groups. ${ }^{60}$

The median number of treatment cycles administered was 3 (range 1-17), and the median time on treatment was 9.3 weeks. $^{61}$ Serious adverse events (SAEs) occurred in $42 \%$ and $47 \%$ in the $2.5 \mathrm{mg} / \mathrm{kg}$ and $3.4 \mathrm{mg} / \mathrm{kg}$ group, and they were considered study-treatment-related in $12 \%$ and $20 \%$ of patients, respectively. Main hematologic grade 3-4 adverse events were thrombocytopenia developing in $22 \%$ of patients receiving Belamaf $2.5 \mathrm{mg} / \mathrm{kg}$ vs $32 \%$ in those $3.4 \mathrm{mg} / \mathrm{kg}$, anemia in $21 \%$ vs $27 \%$, neutropenia in $11 \%$ vs 17\%. Among nonhematologic toxicities, keratopathy (MECs) was the most common adverse event in $72 \%$ of patients in the $2.5 \mathrm{mg} / \mathrm{kg}$ group and $77 \%$ in the $3.4 \mathrm{mg} / \mathrm{kg}$ group. The median time to first MEC was 37 days vs 22.5 days, majority of patients experienced the first event by dose 4 and $48 \%$ and $47 \%$ of patients treated with $2.5 \mathrm{mg} /$ $\mathrm{kg}$ and $3.4 \mathrm{mg} / \mathrm{kg}$, respectively, recovered from the last events at the last follow-up of 13 months. Other ocular events included blurred vision (any grade: $25 \%$ in $2.5 \mathrm{mg} /$ $\mathrm{kg}$ group and $33 \%$ in $3.4 \mathrm{mg} / \mathrm{kg}$ group; grade $3-4: 4 \%$ in both groups); dry eye occurring in $15 \%$ vs $25 \%$ (mostly grade $1-2)$; changes in visual acuity (18\% vs $20 \%$ ), with first episode resolving in $82 \%$ vs $100 \%$ of patients after a median of 21.5 or 23.5 days in $2.5 \mathrm{mg} / \mathrm{kg}$ cohort and $3.4 \mathrm{mg} / \mathrm{kg}$ cohort, respectively. Keratopathy was the main toxicity requiring Belamaf dose delays $(47 \%$ in $2.5 \mathrm{mg} / \mathrm{kg}$ cohort and $53 \%$ in the $3.4 \mathrm{mg} / \mathrm{kg}$ cohort) and dose reduction $(25 \%$ vs $30 \%)$. In contrast, only $1 \%$ vs $3 \%$ of patients permanently discontinued treatment due to ocular toxicities. Other nonhematologic adverse events were not common, and grade 3-4 infections were seen in $7 \%$ and $13 \%$ of patients receiving lower and higher Belamaf dose. The $2.5 \mathrm{mg} / \mathrm{kg}$ was selected as the recommended dose to use in future clinical studies based on similar efficacy data compared with $3.4 \mathrm{mg} / \mathrm{kg}$ but a better safety profile. As regards IRRs, after a median follow-up of 13 months, they occurred in $21 \%$ of patients receiving dose regimen of 
$2.5 \mathrm{mg} / \mathrm{kg}$ and were mostly of grade $1-2$, with no patients developing grade 4-5 IRR. Most patients (90\%) developed the complication during the first cycle, and the incidence of these events declined after that. ${ }^{62}$ Analysis of symptoms and HRQoL of patients enrolled in the DREAMM-2 and receiving the $2.5 \mathrm{mg} / \mathrm{kg}$ dose showed improved pain, physical functioning, and disease symptoms from baseline over time. Of note, improvement in fatigue was seen in $30 \%$ of patients at week $25 .^{63}$

Based on the results of the DREAMM-2 study, belantamab mafodotin was approved by FDA an EMA on August 2020 for the treatment with RRMM who have received at least four prior therapies, including an anti $\mathrm{CD} 38 \mathrm{mAb}$, a PI, and an IMiD. The recommended dosage is $2.5 \mathrm{mg} / \mathrm{kg}$ every three weeks until progression or unacceptable toxicity.

At the last EHA, ASCO, and ASH meetings, post-hoc analyses of specific subpopulations of the DREAMM-2 study have been presented. Patients with mild (defined as eGFR $\geq 60 \leq 90 \mathrm{~mL} / \mathrm{min} / 1.73 \mathrm{~m}^{2}$ ) or moderate (eGFR $\geq$ $30 \leq 60)$ renal impairment achieved a similar ORR compared to patients with normal renal function. However, despite a similar incidence of keratopathy, grade 3-4 thrombocytopenia was more frequent in patients with renal impairment, particularly in patients receiving $3.4 \mathrm{mg} / \mathrm{kg} .{ }^{64}$ Efficacy of Belamaf monotherapy was documented in either standard-risk or high-risk cytogenetics patients with DoR, PFS and probability of a durable response similar in the two groups of patients. ${ }^{65}$ Belamaf was found efficacy in very advanced MM disease as showed by a retrospective analysis comparing the outcome of patients receiving Belamaf $2.5 \mathrm{mg} / \mathrm{kg}$ with 3-6 prior therapies v.s $\geq 7$ prior therapies. ORR was $34 \%$ and $30 \%$ in the first and second group, respectively; median DoR was 11 months vs 13.1 months, median PFS 2.9 vs 2.2 months. No differences in keratopathy, dose delay or dose reduction were reported between the two groups of patients. ${ }^{66}$ Moreover, a recent post-hoc analysis showed that among patients with prolonged treatment interruptions ( $>63$ days), mainly due to keratopathy, $76 \%$ maintained or deepened their response during the delay. ${ }^{67}$

Although the DREAMM-2 study demonstrated the ability of belantamab mafodotin to induce a durable response in heavily pretreated RRMM patients, the company was requested to design a trial comparing Belamaf with another available treatment in this setting of patients, so the ongoing phase III DREAMM-3 trial (Table 2) is evaluating Belamaf vs Pd in approximately in 320 RRMM patients with $\geq 2$ prior lines of therapy. Results from this clinical trial are expected by July $2024 .{ }^{61}$

\section{Pharmacokinetics and Metabolism}

The pharmacokinetics of belantamab mafodotin was assessed by measuring Belamaf, total $\mathrm{mAb}$ (with and without the cytotoxic payload MMAF), and cys-mcMMAF in plasma collected at cycle 1 and 3 of all patients enrolled in the DREAMM- ${ }^{55}$ and DREAMM- ${ }^{58}$ studies. After administration of Belamaf at dose of $2.5 \mathrm{mg} / \mathrm{kg}$, maximal serum concentration $\left(\mathrm{C}_{\max }\right)$ was $42.5 \mu \mathrm{g} / \mathrm{mL}$ after a median of $0.78 \mathrm{~h}\left(\mathrm{t}_{\max }\right)$, mean AUC of $4666 \mu \mathrm{g} . \mathrm{h} / \mathrm{mL}$ and mean $\mathrm{C}_{\text {trough }}$ of $2.4 \mathrm{mg} / \mathrm{mL}$. The mean steady-state volume of distribution of Belamaf was $10.8 \mathrm{~L}$, and it was cleared slowly with a total plasma clearance of $0.92 \mathrm{~L} /$ day and a terminal $t_{1 / 2}$ of 12 days. Over time, clearance was reduced, and $t_{1 / 2}$ was 14 days. ${ }^{68}$

An analysis of population pharmacokinetics and exposure-response for critical endpoints from the DREAMM-2 study showed that time to response but not time to best response was inversely related to Belamaf $\mathrm{C}_{\text {trough }}$. Safety endpoints were strongly associated with exposure, so higher $\mathrm{C}_{\text {trough }}$ was related to the probability of developing ocular exam finding (OEF) and inversely correlated to time to onset. Higher cys-mcMMAF maximum concentration and lower platelet count at baseline were associated with an increased probability of grade 3-4 thrombocytopenia, whereas no factors were found to impact neutropenia and IRRs. Increased likelihood of ocular toxicity and severe thrombocytopenia with higher exposure or dose was not associated with a commensurate improvement in efficacy in the DREAMM-2 study, supporting a monotherapy dose of $2.5 \mathrm{mg} / \mathrm{kg}$ every three weeks in RRMM patients. ${ }^{69}$

\section{ALGONQUIN Study}

The peculiar mechanism of action of Belamaf provides a rationale for combining this $\mathrm{ADC}$ with other active agents used for the treatment of MM as IMiDs, which have shown to exert a pleiotropic effect on a wide range of immune cells as NK cells activation and T cells costimulation. $^{70}$ The multicenter, nonrandomized, ALGONQUIN phase I study, ${ }^{71}$ performed by Canadian Myeloma Research Group, explored safety and efficacy of Belamaf in combination with pomalidomide and dexamethasone in RRMM who had received $\geq 1$ prior line of therapy, lenalidomide refractory, PIs exposed or 
Table 2 Ongoing Clinical Trial with Belantamab Mafodotin

\begin{tabular}{|c|c|c|c|c|}
\hline Trial & Phase & Population & Intervention & Trial ID \\
\hline DREAMM-3 & III & $\begin{array}{l}\text { RRMM with } \geq 2 \text { lines } \\
\text { including PI and } \\
\text { lenalidomide }\end{array}$ & Belamaf vs Pd & NCT04I62210 \\
\hline DREAMM-4 & $1 / I I$ & $\begin{array}{l}\text { RRMM with } \geq 3 \text { lines } \\
\text { including } \mathrm{PI}, \mathrm{IMiD} \text {, and anti } \\
\text { CD38 } \mathrm{mAb}\end{array}$ & Belamaf + pembrolizumab & NCT03848845 \\
\hline DREAMM-5 & $1 / I I$ & $\begin{array}{c}\text { RRMM with } \geq 3 \text { lines } \\
\text { including } \mathrm{PI}, \mathrm{IMiD} \text {, and anti } \\
\text { CD38 } \mathrm{mAb}\end{array}$ & Belamaf + innovative drugs & NCT04I 26200 \\
\hline DREAMM-6 & $\mathrm{I} / \mathrm{II}$ & $\begin{array}{l}\text { RRMM with } \geq 1 \text { prior } \\
\text { therapy }\end{array}$ & Belamaf-Rd or Belamaf-Vd & NCT0354428I \\
\hline DEAMM-7 & III & $\begin{array}{l}\text { RRMM with } \geq 1 \text { prior } \\
\text { therapy }\end{array}$ & Belamaf-Vd vs DVd & NCT042246047 \\
\hline DEAMM-8 & III & $\begin{array}{l}\text { RRMM with } \geq \text { I prior } \\
\text { therapy including } \\
\text { lenalidomide }\end{array}$ & Belamaf-Pd vs PVd & NCT04484623 \\
\hline DREAMM-9 & I & $\begin{array}{l}\text { Transplant ineligible } \\
\text { NDMM }\end{array}$ & Belamaf-VRd followed by Belamaf-Rd & NCT0409II 26 \\
\hline DREAMM-I2 & $\mathrm{I}$ & $\begin{array}{l}\text { Renal impairment } \\
\text { RRMM with } \geq 2 \text { lines } \\
\text { including PI and IMiD, }\end{array}$ & Belamaf & NCT04398745 \\
\hline DREAMM-I3 & I & $\begin{array}{l}\text { Hepatic impairment } \\
\text { RRMM with } \geq 2 \text { lines } \\
\text { including } \mathrm{PI} \text { and } \mathrm{IMiD} \text {, }\end{array}$ & Belamaf & NCT04398680 \\
\hline $\begin{array}{l}\text { Study of belamaf as pre-and post-autologous } \\
\text { stem cell transplant and maintenance for } \\
\text { MM }\end{array}$ & I & Transplant eligible NDMM & $\begin{array}{c}\text { Belamaf pre- (day }-42) \text { and post- ASCT } \\
\text { for } 2 \text { years }\end{array}$ & NCT04680468 \\
\hline $\begin{array}{l}\text { Blmf, lenalidomide, and dexamethasone in } \\
\text { transplant-ineligible patients with newly } \\
\text { diagnosed multiple myeloma (BelaRd) }\end{array}$ & $\mid / I I$ & $\begin{array}{l}\text { Transplant ineligible } \\
\text { NDMM }\end{array}$ & BelaRd & NCT04808037 \\
\hline $\begin{array}{l}\text { Belantamab mafodotin in newly diagnosed } \\
\text { transplant eligible multiple myeloma patients }\end{array}$ & II & Transplant eligible NDMM & $\begin{array}{c}\text { Bela-VRd followed by ASCT, } \\
\text { consolidation with Belamaf and VRd, } \\
\text { maintenance with lenalidomide and } \\
\text { Belamaf }\end{array}$ & NCT04802356 \\
\hline $\begin{array}{l}\text { Characterization of corneal epithelial } \\
\text { changes in participants treated with } \\
\text { belantamab mafodotin }\end{array}$ & III & $\begin{array}{l}\text { RRMM who have received } \\
\text { or are receiving belamaf } \\
\text { and with keratopathy }\end{array}$ & $\begin{array}{l}\text { Impression cytology or superficial } \\
\text { keratectomy }\end{array}$ & NCT045549363 \\
\hline
\end{tabular}

Abbreviations: Pd, pomalidomide, dexamethasone; Rd, lenalidomide, dexamethasone; Vd, bortezomib, dexamethasone; DVd, daratumumab, bortezomib, dexamethasone; PVd, pomalidomide, bortezomib, dexamethasone; VRd, bortezomib, lenalidomide, dexamethasone; RRMM, relapsed/refractory multiple myeloma; NDMM, newly diagnosed multiple myeloma.

refractory, and pomalidomide naïve. In Part 1, dosefinding patients received pomalidomide $4 \mathrm{mg}$ days $1-21$, dexamethasone $40 \mathrm{mg}$ weekly (20 $\mathrm{mg}$ in patients older than 75 years), and belamaf single (1.92 or $2.5 \mathrm{mg}$ / $\mathrm{kg}$ day 1 ) or split (2.5 or $3.4 \mathrm{mg} / \mathrm{kg}$ spit on days 1 and 8 ) every four weeks. The primary endpoint of this part was to identify the MTD and RP2D to use in Part 2, which aimed to evaluate the ORR of the combination. At the 
presentation of the study at the last ASH meeting, the number of patients enrolled in the dose-finding phase was 35 with a median age of 64 years (range 36-81) and a median of 3 prior lines of therapy (range 1-5). Seventy-three percent of patients were lenalidomide and PIs refractory, whereas $35 \%$ were triple-refractory (lenalidomide, PIs, and daratumumab). MTD was established to be Belamaf $2.5 \mathrm{mg} / \mathrm{kg}$ (day 1) and $2.5 \mathrm{mg} / \mathrm{kg}$ splitdosing $(1,25 \mathrm{mg} / \mathrm{kg}$ days 1 and 8$)$ every four weeks in combination with standard doses of pomalidomide and dexamethasone (Pd). Grade 3-4 hematologic adverse events consisted of neutropenia (40.5\%) and thrombocytopenia (32.4\%). Grade 3-4 keratopathy occurred in 51\% of patients and decreased visual acuity in $16 \%$. IRRs developed in $29 \%$ of patients, but they were mainly of grade 1-2. Overall, at least $88 \%$ of patients achieved at least PR $(\geq$ VGPR $=68 \%$; CR $=20.6 \%)$ and, after a median follow-up of 7.8 months, median PFS was not reached. Efficacy was also documented in triplet refractory MM patients since ORR was $100 \%$, with $72 \%$ of patients obtaining at least VGPR. However, a single dose of $1.92 \mathrm{mg} / \mathrm{kg}$ in combination with $\mathrm{Pd}$ was found to be the best in terms of efficacy and safety profile since ORR was $82 \%$, at least VGPR $64 \%$, median PFS 14.1 months, and grade 3-4 keratopathy 25\%, showing that Belamaf-Pd compares favorably to other pomalidomide-based triplets as pomalidomide, Bortezomib, dexamethasone (PVd: median PFS 11.2 months) ${ }^{72}$ elotuzumab, pomalidomide, dexamethasone (Elo-Pd: median PFS 10.3 months), ${ }^{13}$ daratumumab, pomalidomide, dexamethasone (DPd: median PFS 12.4 months), ${ }^{19}$ isatuximab, pomalidomide, dexamethasone (Isa-Pd: median PFS 11.5 months) ${ }^{25}$ and, finally, selinexor-Pd (median PFS 10.4 months). ${ }^{73}$

\section{DREAMM-4 Study}

Belamaf was evaluated in association with pembrolizumab in the phase I/II DREAMM-4 study ${ }^{74}$ in RRMM patients with $\geq 3$ prior lines of therapy, including antiCD38 mAb. The rationale of this combination is that PD-1 inhibitors may augment immune responses to Belamaf-induced immunogenic cell death. In the doseescalation part of the study, patients received Belamaf $2.5 \mathrm{mg} / \mathrm{kg}$ or $3.4 \mathrm{mg} / \mathrm{kg}$ plus pembrolizumab $200 \mathrm{mg}$ iv every three weeks. A total of 13 patients with a median age of 71 years (range 50-81) and a median number of prior therapies of 5 (3-13) were enrolled in this study phase. The ORR was $67 \%$ and $43 \%$ in the $2.5 \mathrm{mg} / \mathrm{kg}$ and $3.4 \mathrm{mg} / \mathrm{kg}$ cohort, respectively. Ocular toxicity represented the most common AEs: any grade keratopathy (with or without symptoms) was reported in $83 \%$ and $57 \%$, being of grade $3-4$ in $33 \%$ and 0 patients, respectively. Both blurred vision and dry eye were seen in 50\% of patients in the $2.5 \mathrm{mg} / \mathrm{kg}$ group, whereas they were $14 \%$ in $3.4 \mathrm{mg} / \mathrm{kg}$. Of note, adding pembrolizumab to Belamaf increased the incidence of grade 3-4 pneumonia, occurring in $17 \%$ of patients receiving Belamaf $2.5 \mathrm{mg} / \mathrm{kg}$ and $29 \%$ in those $3.4 \mathrm{mg} / \mathrm{kg}$. Based on the results of Part 1 dose-escalation, patients enrolled in the Part 2 cohort expansion are receiving Belamaf at the dose of $2.5 \mathrm{mg} /$ $\mathrm{kg}$ every three weeks.

\section{Other Ongoing Clinical Trials with Belantamab Mafodotin}

In the DREAMM-5 Platform trial, ${ }^{75}$ Belamaf has been evaluated in combination with other anti-myeloma agents in RRMM patients with $\geq 3$ prior lines of therapy. The sub-studies currently open or opening soon to enrollment include Belamaf combined with GSK3174998, a humanized wild-type IgG1 anti-OX40 agonist antibody, binding to co-stimulatory OX40 receptor expressed on activated CD4+ and CD8+ cells enhancing immune-mediated anti-cancer activity (sub-study 1); in the sub-study 2, Belamaf is evaluated with feladilimab (GSK3359609) a humanized anti-ICOS agonist antibody binding to ICOS-expressing $\mathrm{T}$ cells; nirogacestat, a selective $\gamma$-secretase inhibitor able to increase the cellsurface level and availability of BCMA, is under evaluation with Belamaf in the sub-study 3; sub-study 4 assesses Belamaf with dostarlimab, a humanized antiPD-1 antibody and, finally, sub-study 5 evaluates Belamaf pus isatuximab.

DREAMM- $6^{76}$ is an ongoing, two-part, two-arm, open-label, phase I/II study of Belamaf in combination with lenalidomide-dexamethasone (Rd) (arm A) or bortezomib-dexamethasone (Vd) ( $\mathrm{arm} B$ ) in patients with $\geq 1$ prior line of therapy (bortezomib-refractory patients are not excluded). At the last ASH Meeting, preliminary results of 18 patients enrolled in arm B have been presented. Patients had received a median of 3 prior lines of therapy (range 1-11) and received Belamaf $2.5 \mathrm{mg} / \mathrm{kg}$ on day 1 , bortezomib $1.3 \mathrm{mg} / \mathrm{m}^{2}$ on days $1,4,8,11$ of cycles $1-8$, and dexamethasone $20 \mathrm{mg}$ on days $1,2,4,5$, $8,9,11,12$. After eight cycles, patients could receive Belamaf monotherapy until progression or unacceptable 
toxicity. All patients experienced keratopathy, being of grade 3 in $61 \%$ of them, whereas any grade blurred vision and dry eye were seen in $67 \%$ and $22 \%$ of patients, respectively. Grade 3-4 thrombocytopenia occurred in $78 \%$ and peripheral neuropathy, grade $1-2$, in $33 \%$ of patients. As regard activity, ORR was $78 \%$ and $\geq$ VGPR $67 \%$, and, after a median of 18.2 weeks on treatment, median DoR was not reached.

DREAMM $-7^{77}$ is an ongoing phase III trial comparing Belamaf, Bortezomib, dexamethasone (B-Vd) to daratumumab, Bortezomib, dexamethasone (D-Vd) in RRMM patients with $\geq 1$ prior lines of therapy. The primary endpoint is PFS. The key secondary endpoint is MRD, as assessed by next-generation sequencing. As of November 2020, 108 patients have been enrolled.

Another phase III trial (DREAMM-8) ${ }^{78}$ is studying Belamaf, pomalidomide, dexamethasone (B-Pd) vs pomalidomide, Bortezomib, dexamethasone (PVd) in RRMM patients with $\geq 1$ prior lines of therapy (including lenalidomide). The primary objective is PFS, and the key secondary is MRD negativity rate. The trial started in October 2020 and aimed to enroll 450 patients worldwide.

In Table 2, we summarized ongoing clinical trials with belantamab mafodotin.

\section{Toxicities of Belantamab Mafodotin}

Ocular toxicity is the most important AE of Belamaf: ocular events included keratopathy (microcyst-like epithelial changes [MECs]: an eye exam finding with/without symptoms), best-corrected visual acuity (BCVA) changes, and symptoms (blurred vision and dry eye). ADC toxicities could be explained by on-target or off-target mechanisms: given the majority of the proteins targeted by these agents are not expressed in the cornea (eg, BCMA), the MECs may primarily represent an off-target mechanism. Farroq et $\mathrm{al}^{79}$ hypothesized that Belamaf could reach corneal cells through vascularized part of the limbus or through the tear film because Belamaf has been detected in rabbit tears. Belamaf can be internalized through the process called micropinocytosis in the limbal epithelial stem cells, inducing their apoptosis and starting the migration of the still alive epithelial cells toward the peripheral cornea. As long as these corneal cells are in the peripheral zone of the cornea, MECs can be identified at the slit lamp images but without any ocular symptoms. When the migration reaches the central cornea and crosses the visual axis, blurred vision and BCVA changes are reported. Over time, the migration of new corneal epithelial cells to replace dead cells that are extruded, allows the resolution of MECs and symptoms. Median timing of resolution reflects the timing of corneal proliferation, from 14 days to several weeks. These migrating Belamaf-containing cells can be visualized by IVCM (in vivo confocal microscopy) as hyperreflective opacities. ${ }^{79}$

Published results of Phase 2 DREAMM-2 study ${ }^{58}$ showed that the most common grade 3-4 AE was keratopathy ( $27 \%$ and $21 \%$ in the two cohorts, respectively). In patients receiving Belamaf single-agent $2.5 \mathrm{mg} / \mathrm{kg}, 72 \%$ of patients experienced MECs, but fewer patients (56\%) had symptoms and/or $\mathrm{a} \geq 2$ lines BCVA decline. Events led to treatment discontinuation in $3 \%$ of patients: $1 \%$ each due to MECs, blurred vision, and reduced BCVA. The median time to the onset of ocular AEs with Belamaf $2.5 \mathrm{mg} / \mathrm{kg}$ were 37 days for MECs, 64 for BCVA changes, 52 for blurred vision, and 42 for dry eyes. The median time to the duration of these were 87 days for MECs, 33 for BCVA changes, 43 for blurred vision, and 39 for dry eyes. The examination of the outcome of ocular events at the first occurrence showed that $77 \%$ of patients recovered from their MEC event, and $82 \%$ of patients recovered from their BCVA decline (measured as the change in BCVA worse than $20 / 50$ in the better-seeing eye). Data from the last follow-up showed that $48 \%$ of patients recovered from their MEC event, and $82 \%$ of them recovered from their BCVA decline. ${ }^{58,80,81}$

Ferron-Brady et $\mathrm{al}^{82}$ demonstrated that higher Belamaf $\mathrm{C}_{\text {trough }}$ (the predicted concentration on day 21 at the end of the first cycle) was associated with great probability of grade $\geq 2$ or $\geq 3$ corneal events or with an earlier onset of them, but not with the probability of blurred vision, dry eye or decline of BCVA than 20/50. History of dry eye was associated with a higher probability of any grade blurred vision, while the initial presence of keratopathy increased the probability of grade $\geq 2$ blurred vision. It is not recommended to administer Belamaf to patients with keratopathy at baseline.

As for the management of ocular toxicity, the ocular sub-study of DREAMM- $2^{83}$ demonstrated that prophylaxis with corticosteroid tears was ineffective in preventing ocular toxicity. The use of cooling eye mask or vasoconstrictors are recommended to minimize ocular exposure to the drug, but their benefit is unclear. The only helpful intervention to block ocular toxicity is dose delay or dose reductions to allow time for replacement of corneal epithelial cells. Ongoing studies are evaluating alternative dose reduction strategies, including split dosing or less 
frequent dosing. Cohen et $\mathrm{al}^{67}$ showed that treatment delay $>63$ days did not negatively impact the response to therapy: they examined 16 patients in the DREAMM-2 trial who experienced dose delay $>63$ days. Fourteen of them $(88 \%)$ continued to experience a clinical benefit during the first prolonged delay: 38\% deepened their response during the delay, 38\% maintained the same response, and $13 \%$ had increasing paraprotein without criteria for disease progression. Popat et $\mathrm{al}^{63}$ demonstrated that patients showed a general improvement in fatigue during the treatment period and, despite ocular AEs, their quality of life did not worsen over time. Lin et $\mathrm{al}^{84}$ studied imipramine as a new potential drug against micropinocytosis in cellular and biological systems, and it could be a new approach to fight against ocular toxicity of Belamaf. Given the process of micropinocytosis could also be involved in Belamafrelated thrombocytopenia, the identification of useable micropinocytosis inhibitors should be an active research topic.

Overall, given the frequency of ocular AEs with Belamaf, it is very important to better understand its pathogenetic mechanisms and clinical features in order to make the most suitable treatment judgments. Multidisciplinary management of Belamaf therapy is crucial and ophthalmologic evaluation, at baseline (including visual acuity measurement and slit-lamp microscopy) up to 3 weeks before the first Belamaf administration, prior to each cycle (up to 2 weeks before) and then whenever clinically recommended, is mandatory.

The targeted delivery of chemotherapy with ADCs should have reduced toxicities compared to systemic chemotherapy, but auristatin is associated with haematological toxicity when attached to non-cleavable linkers. Toxicity can occur due to the expression of antigens on normal tissues (on-target), inadequate linker stability leading to systemic release of the payload, or off-target uptake of the linker-payload compound. Apart from ocular toxicity, thrombocytopenia was the most relevant side effect, explained through the same pathogenetic mechanism as the corneal toxicity via the apoptosis of megakaryocyte progenitor cells. In DREAMM-1 trial ${ }^{55}$ grade, 3-4 thrombocytopenia and anemia were $35 \%$ and $17 \%$, respectively. Grade 3-4 thrombocytopenia occurred in $22 \%$ of patients receiving Belamaf $2.5 \mathrm{mg} / \mathrm{kg}$ vs $32 \%$ in the $3.4 \mathrm{mg} / \mathrm{kg}$ group of DREAMM-2 ${ }^{58}$ study (anemia $21 \%$ and $27 \%$, respectively). Thrombocytopenia was reported in $78 \%$ of patients in the DREAMM- 6 trial, ${ }^{76}$ leading to dose discontinuation in $33 \%$ of patients and dose delay in $39 \%$.
IRRs occurred in $21 \%$ of patients in the $2.5 \mathrm{mg} / \mathrm{kg}$ cohort and $16 \%$ in the $3.4 \mathrm{mg} / \mathrm{kg}$ group of the DREAMM-2 trial. $^{58}$ Most of them were limited to the first infusion, and nearly all were grade $1-2$. Grade $\geq 3$ IRRs during the first dose were 3\%. Premedication was not required by the protocol, and when it was used (1/4 of all patients), it did not proportionately decrease the incidence of IRRs of these patients compared to the overall study population. Grade 2 IRRs were reported in $17 \%$ of patients in the DREAMM-6 trial without any dose modifications or discontinuations.

Other minor toxicities were pneumonia, hypertension, GGT increase, AST increase, hypercalcemia, and fatigue.

\section{Discussion}

Several drugs are now available for the treatment of MM, making treatment selection and sequencing of compounds a complex process. Clonal heterogeneity and complexity of $\mathrm{MM}$ require multiple drug associations through all disease phases to eliminate all clonal cells and long-term or continuous therapy to keep tumor cells under control. Therefore, most patients quickly become refractory to multiple drugs in their disease history, so agents with novel mechanisms of action are needed for these patients. Belamaf is the first-in-class anti-BCMA ADC approved for RRMM with a peculiar mechanism of action that makes it unique in the therapeutic landscape of MM. DREAMM-1 ${ }^{55,57}$ and, even more, DREAMM-2 ${ }^{58}$ study established that a single drug with these characteristics can be effective in patients with very advanced MM patients in whom deep response (20\% VGPR) and longterm remission duration (around one year) were achieved. The higher response rates observed in the dose-expansion DREAMM-1 ${ }^{57}$ study can be attributed to the lower percentage of patients refractory to daratumumab (40\% vs $100 \%$ in the $2.5 \mathrm{mg} / \mathrm{kg}$ group of DREAMM-2 study ${ }^{58}$ ) as well as to fewer patients with more than four prior lines of therapy, a very difficult-to-treat population (57\% vs $84 \%$ ). These results would have never been achieved with conventional chemotherapies or more recent therapies such as selinexor that, combined with dexamethasone, led to a similar response rate but with a shorter duration (median five months). ${ }^{28}$ Moreover, despite better ORR and median PFS, $84 \%$ of patients in the SVd arm of BOSTON trial $^{27}$ had received 1 or 2 prior lines of therapy, and only $6 \%$ had been exposed to daratumumab. The response with Belamaf was maintained or improved in patients who hold therapy for toxicity, suggesting a disease control by the 
immune system and the possibility of dose-intensity reduction or alternative schedules. The same group of triple- or penta-refractory patients is the population on active investigation for other immunotherapies as bispecific antibodies and CAR T cell therapies with which an impressive rate of deep response has been obtained. ${ }^{33,34}$ ORRs obtained with bispecific antibodies as teclistamab, and CAR T cell therapy as idecabtagene vicleucel in triple-refractory MM patients are very impressive, but longer follow-up is required to see whether the response can last over time, so Belamaf, besides selinexor, represents now one of the most appropriate therapeutic options in very advanced MM as suggested by the most recent EHA-ESMO guidelines. ${ }^{85}$ Moreover, the activity of CAR T cells and bispecific antibodies depend on T-cell "fitness", and it could be reduced with the loss of BCMA expression due to prior BCMA-directed therapy such as Belamaf. Both bispecific antibodies and CAR $T$ cell therapies require hospitalization in accredited centers with ICU and neurology services due to possible life-threatening toxicity such as CRS (cytokine release syndrome) and ICANS (immune effector cell-associated neurotoxicity syndrome), never seen with Belamaf. In contrast with CAR $T$ cells and bispecific antibodies, Belamaf could be prescribed in the elderly. In fact, among patients enrolled in the DREAMM2 trial, ${ }^{58} 59 \%$ were 65 years or older, whereas, in the KarMMa trial, ${ }^{35}$ using idecabtagene vicleucel, a CAR $\mathrm{T}$ cell therapy, $35 \%$ of patients were $\geq 65$ years old. As shown above, grade 3-4 neutropenia, thrombocytopenia, and infections occurred in $22 \%, 11 \%$, and $7 \%$, respectively, of patients receiving Belamaf $2.5 \mathrm{mg} / \mathrm{kg}$ vs $89 \%$, $52 \%$, and $22 \%$ seen in patients treated with CAR T cell therapy. Moreover, $84 \%$ of patients receiving idecabtagene vicleucel developed CRS and 18\% neurologic effects. CAR $\mathrm{T}$ cell therapies require conditioning chemotherapy and a personalized manufacturing process that could be prohibitive for rapid progression disease, whereas Belamaf and bispecific antibodies are "off-the-shelf" and immediately available. Belamaf, due to its targeted cytotoxicity, acts independently on $\mathrm{T}$ cells fitness. In contrast, the outcome of patients treated with both bispecific antibodies and CAR T cells are strictly dependent on host immune system performance, that has deteriorated in the elderly population. Moreover, subgroups analyses of patients treated with Belamaf monotherapy did not demonstrate excessive toxicity in patients with renal or hepatic insufficiency, high-risk or extramedullary disease, although activity seems to be lower in patients refractory to daratumumab.
Several studies with Belamaf in combination with IMiDs, PIs, and other compounds are ongoing in late and early disease relapse since Belamaf toxicity seems to be not cumulative with other drugs while increasing effectiveness is expected. The combination of Belamaf with pomalidomide and dexamethasone showed good response in advanced RRMM ${ }^{71}$, but ongoing trials (Table 2) are assessing Belamaf in combination with $\mathrm{Rd}, \mathrm{Pd}$, or $\mathrm{Vd}$ in the first relapse or in NDMM in combination with VRD, and they could establish the role of this agent in the management of early MM.

Unfortunately, treatment with Belamaf may cause very peculiar toxicity. Ocular toxicity, until now an unknown side effect in MM therapy, can be seen after therapy with Belamaf. Corneal toxicity is very common, although it is mild and has no consequences for the patients in most cases. However, about a quarter of cases have significant, albeit reversible, visual disturbances that require reduction or discontinuation of therapy, rarely definitive discontinuation. This side effect is little known by haematologists. Therefore, when it occurs, it requires active collaboration with ophthalmologists to timely diagnose corneal/MEC changes and intervene in a timely fashion, which could be just dose delay or reduction and does not necessarily need an ophthalmic procedure. This issue will become even more important when Belamaf is used earlier in the course of MM rather than in patients with very advanced disease for whom alternatives are certainly fewer. However, Belamaf-associated ocular toxicity is the subject of very active research that tends to better understand the mechanism that induces toxicity leading to the discovery of possible antidotes, but at the moment, reduction of dose-intensity or alternative schedules are the main ways of research.

\section{Conclusion}

Belantamab mafodotin is the first BCMA-targeted immunotherapy to be approved for the treatment of advanced RRMM, representing hope for patients who have become refractory to all available classes of drugs. It showed efficacy as a single agent, but several ongoing trials are evaluating Belamaf in combination with all available agents and also with experimental molecules. Ocular toxicity is of concern, but further multidisciplinary cooperation will help to likely make this adverse event predictable and easy to manage. 


\section{Disclosure}

Massimo Offidani received honoraria from and served as an advisor for Glaxo-Smith-Kline, during the conduct of the study.

The authors report no other potential conflicts of interest for this work.

\section{References}

1. National Cancer Institute. Cancer stat facts: myeloma. Available from: http://seer.cancer.gov/statfacts/htlm/mulmy.htlm.

2. Anderson KC. Progress and paradigms in multiple myeloma. Clin Cancer Res. 2016;22:5419-5427.

3. Kumar SK, Rajkumar SV, Dispenzieri A, et al. Improved survival in multiple myeloma and the impact of novel therapies. Blood. 2008;111:2516-2520.

4. Kumar SK, Dispenzieri A, Lacy MQ, et al. Continued improvement in survival in multiple myeloma: changes in early mortality and outcomes in older patients. Leukemia. 2014;28:1122-1128.

5. Tacchetti P, Patriarca F, Petrucci MT, et al. A triplet bortezomib- and immunomodulator-based therapy before and after double ASCT improves overall survival of newly diagnosed MM patients: final analysis of Phase 3 GIMEMA-MMY-3006 study. Hemasphere. 2018;2(S1):abstractS105.

6. Nandakumar B, Binder M, Dispenzieri A, et al. Continued improvement in survival in multiple myeloma, including high-risk patients. J Clin Oncol. 2019;37(suppl):abstract 8039.

7. Kumar SK, Therneau TM, Gertz MA, et al. Clinical course of patients with relapsed multiple myeloma. Mayo Clin Proc. 2004;79:867-874.

8. Gandhi UH, Cornell RF, Lakshman A, et al. Outcomes of patients with multiple myeloma refractory to CD38-targeted monoclonal antibody therapy. Leukemia. 2019;33:2266-2275.

9. Coiffier B, Thieblemont C, Van Den Neste E, et al. Long-term outcome of patients in the LNH-98.5 trial, the first randomized study comparing rituximab-CHOP to standard CHOP chemotherapy in DLBCL patients: a study by the Groupe d'Etudes des Lymphomes de l'Adulte. Blood. 2010;116:2040-2045.

10. Connors JM, Jurczak W, Straus DJ, et al. Brentuximab vedotin with chemotherapy for stage III or IV Hodgkin's lymphoma. $N$ Engl $J$ Med. 2018;378(4):331-344. doi:10.1056/NEJMoa1708984

11. Offidani M, Corvatta L. A review discussing elotuzumab and its use in the second-line plus treatment of multiple myeloma. Future Oncol. 2018;14:319-329.

12. Lonial S, Dimopoulos MA, Palumbo A, et al. Elotuzumab therapy for relapsed or refractory multiple myeloma. $N$ Engl $J$ Med. 2015;373:621-631.

13. Dimopoulos MA, Dytfeld D, Grosicki S, et al. Elotuzumab plus pomalidomide and dexamethasone for multiple myeloma. $N$ Engl J Med. 2018;379:1811-1822.

14. Nooka AK, Kaufman JL, Hofmeister CC, et al. Daratumumab in multiple myeloma. Cancer. 2019;125:2364-2382.

15. Lokhorst HM, Plesner T, Laubach JP, et al. Targeting CD38 with daratumumab monotherapy in multiple myeloma. New Engl J Med. 2015;373:1207-1219.

16. Lonial S, Weiss BM, Usmani SZ, et al. Daratumumab monotherapy in patients with treatment-refractory multiple myeloma (SIRIUS): an open-label, randomised, phase 2 trial. Lancet. 2016;387:1551-1560.

17. Dimopoulos MA, Oriol A, Nahi H, et al. Daratumumab, lenalidomide, and dexamethasone for multiple myeloma. New Engl J Med. 2016;375:1319-1331.
18. Palumbo A, Chanan-Khan A, Weisel K, et al. Daratumumab, Bortezomib, and dexamethasone for multiple myeloma. New Engl J Med. 2016;375:754-766.

19. Dimopoulos MA, Terpos E, Boccadoro M, et al. Apollo: phase 3 randomized study of subcutaneous daratumumab plus pomalidomide and dexamethasone (D-Pd) versus pomalidomide and dexamethasone (Pd) alone in patients with relapsed/refractory multiple myeloma. Blood. 2020;136(Suppl 1):abstract 412.

20. Dimopoulos M, Quach H, Mateos M-V, et al. Carfilzomib, dexamethasone, and daratumumab versus Carfilzomib and dexamethasone for patients with relapsed or refractory multiple myeloma (CANDOR): results from a randomized, multicenter, open-label, phase 3 trial. Lancet. 2020;396:186-197.

21. Moreau P, Attal M, Hulin C, et al. Bortezomib, thalidomide, and dexamethasone with or without daratumumab before and after autologous stem-cell transplantation for newly diagnosed multiple myeloma (CSSIOPEIA): a randomised, open-label, phase 3 study. Lancet. 2019;394:29-38.

22. Mateos MV, Dimopoulos MA, Cavo M, et al. Daratumumab plus Bortezomib, melphalan, and prednisone for untreated myeloma. N Engl J Med. 2018;378:518-528.

23. Facon T, Kumar S, Plesner T, et al. Daratumumab plus lenalidomide and dexamethasone for untreated myeloma (MAIA). $N$ Engl J Med. 2019;380:2104-2105.

24. Feng X, Zhang L, Acharya C, et al. Targeting CD38 suppresses induction and function of $t$ regulatory cells to mitigate immunosuppression in multiple myeloma. Clin Cancer Res. 2017;23:4290-4300.

25. Attal M, Richardson PG, Rajkumar SV, et al. Isatuximab plus pomalidomide and low-dose dexamethasone versus pomalidomide and low-dose dexamethasone in patients with relapsed and refractory multiple myeloma (ICARIA-MM): a randomised, multicentre, open-label, phase 3 study. Lancet. 2019;394:2096-2107.

26. Gravina GL, Senapedis W, McCauley D, et al. Nucleo-cytoplasmic transport as a therapeutic target of cancer. J Hematol Oncol. 2014;7:85.

27. Grosicki S, Simonova M, Spicka I, et al. Once-per-week selinexor, Bortezomib, and dexamethasone versus twice-per-week Bortezomib and dexamethasone in patients with multiple myeloma (BOSTON): a randomized, open-label, phase 3 trial. Lancet. 2020;396:1563-1573.

28. Chari A, Vogl DT, Gavriatopoulou M, et al. Oral selinexor-dexamethasone for triple-class refractory multiple myeloma. N Engl J Med. 2019;381:727-738.

29. Chauhan D, Ray A, Viktorsson K, et al. In vitro and in vivo antitumor activity of a novel alkylating agent, melphalan-flufenamide, against multiple myeloma cells. Clin Cancer Res. 2013;19:3019-3031.

30. Richardson PG, Oriol A, Larocca A, et al. HORIZON (OP-106): melflufen plus dexamethasone in relapsed/refractory multiple myeloma refractory to pomalidomide and/or anti-CD38 monoclonal antibody-primary and subgroup analysis. Hemasphere. 2020;4(S1): abstract EP 945.

31. Bjorklund CC, Kang J, Amatangelo M, et al. Iberdomide (CC-220) is a potent cereblon E3 ligase modulator with antitumor and immunostimulatory activities in lenalidomide- and pomalidomide-resistant multiple myeloma cells with dysregulated CRBN. Leukemia. 2020;34:1197-1201.

32. Kumar SK, Harrison SJ, Cavo M, et al. Venetoclax or placebo in combination with Bortezomib and dexamethasone in patients with relapsed or refractory multiple myeloma (BELLINI): a randomized, double-blind, multicenter, phase 3 trial. Lancet Oncol. 2020;21:1630-1642.

33. Lancman G, Richter J, Chari A. Bispecific, trispecific, and other novel immune treatments in myeloma. Hematology. 2020;264-271.

34. Mikkilinemi L, Kochenderfer JN. Chimeric antigen receptor T-cell therapies for multiple myeloma. Blood. 2017;130:2594-2602. 
35. Munshi NC, Anderson LD, Sha N, et al. Idecabtagene vicleucel in relapsed and refractory multiple myeloma. $N$ Engl $J$ Med. 2021;384:705-716.

36. Garfall AL, Usmani SZ, Mateos M-V, et al. Updatedp Phase 1 results of teclistamab, a B-Cell Maturation Antigen (BCMA) x CD3 bispecific antibody, in relapsed and/or refractory multiple myeloma (RRMM). Blood. 2020;136(Suppl 1):abstract 27.

37. Tai YT, Anderson KC. Antibody-based therapies in multiple myeloma. Bone Marrow Res. 2011;2011:924058.

38. Tamura H. Immunopathogenesis and immunotherapy of multiple myeloma. Int J Hematol. 2018;107:278-285.

39. Neri P, Alberge J-B, Maity R, et al. Expansion of effector memory $\mathrm{CD} 27+\mathrm{T}$ cells and tolerogenic type 2 classical dendritic cells regulate myeloma patients' sensitivity to daratumumab and IMiDS. Clin Lymph Myeloma Leuk. 2019;19:e29-e30.

40. Nahi H, Chrobok M, Gran C, et al. Infectious complications and NK cell depletion following daratumumab treatment of Multiple Myeloma. PloSOne. 2019;14:e0211927.

41. Tsuchikama K, Zhiqiang A. Antibody-drug conjugates: recent advances in conjugation and linker chemistries. Protein Cell. 2018;9:33-46.

42. Madry C, Laabi Y, Callebaut I, et al. The characterization of murine BCMA gene defines it as a new member of the tumor necrosis factor receptor superfamily. Int Immunol. 1998;10:1693-1702.

43. Rennert P, Schneider P, Cachero TG, et al. A soluble form of B cell maturation antigen, a receptor for the tumor necrosis factor family member APRIL, inhibits tumor cell growth. $J$ Exp Med. 2000;192:1677-1683.

44. Tai Y-T, Acharya C, An G, et al. APRIL and BCMA promote human multiple myeloma growth and immunosuppression in the bone marrow microenvironment. Blood. 2016;127:3225-3236.

45. Sanchez E, Li M, Kitto A, et al. Serum B-cell maturation antigen is elevated in multiple myeloma and correlates with disease status and survival. Br J Haematol. 2012;158:727-738.

46. Dispenzieri A, Soof CM, Rajkumar V, et al. Serum BCMA levels to predict outcomes for patients with MGUS and smoldering multiple myeloma (SMM). J Clin Oncol. 2019;37(15 Suppl):abstract 8020

47. Ghermezi M, Li M, Vardanyan S, et al. Serum B-cell maturation antigen: a novel biomarker to predict outcomes for multiple myeloma patients. Haematologica. 2017;102:785-795.

48. Tolcher AW. The evolution of antibody-drug conjugates: a positive inflexion point. Am Soc Clin Oncol Educ Book. 2020;40:1-8.

49. Lu J, Jiang F, $\mathrm{Lu} \mathrm{A}$, et al. Linkers having a crucial role in antibody-drug conjugates. Int J Mol Sci. 2016;17:561.

50. Yu B, Liu D. Antibody-drug conjugates in clinical trials for lymphoid malignancies and multiple myeloma. J Hematol Oncol. 2019;12:94.

51. Herrera AF, Molina A. Investigational antibody-drug conjugates for treatment of B-lineage malignancies. Clin Lymphoma Myeloma Leuk. 2018;18:452-468.

52. GlaxoSmithKline. BLENREP (belantamab mafodotin-blmf): US prescribing information. 2020. https://www.accessdata.fda.gov/. Accessed May 24, 2021.

53. Tai YT, Mayes PA, Acharya C, et al. novel anti-B-cell maturation antigen antibody-drug conjugate (GSK2857916) selectively induces killing of multiple myeloma. Blood. 2014;123:3128-3138.

54. Montes de Oca R, Bhattacharya S, Vitali N, et al. The anti-BCMA antibody-drug conjugate GSK2857916 drivers immunogenic cell death and immune-mediated antitumor responses, and in combination with an OX40 agonist potentiates in vivo activity. HemaShere. 2019;3(S2):abstractPF558.

55. Trudel S, Lendvai N, Popat R, et al. Targeting B-cell maturation antigen with GSK2857916 antibody-drug conjugate in relapsed or refractory multiple myeloma (BMA117159): a dose escalation and expansion phase 1 trial. Lancet Oncol. 2018;19:1641-1653.
56. Eaton JS, Miller PE, Mannis MJ, et al. Ocular adverse events associated with antibody-drug conjugates in human clinical trials. $J$ Ocul Pharmacol Ther. 2015;31:589-604.

57. Trudel S, Lendvai N, Popat R, et al. Antibody-drug conjugate, GSK2857916, in relapsed/refractory multiple myeloma: an update on safety and efficacy from dose expansion phase I study. Blood Cancer J. 2019;9:37.

58. Lonial S, Lee HC, Badros A, et al. Belantamab mafodotin for relapsed or refractory multiple myeloma (DREAMM-2): a two-arm, randomised, open-label, phase 2 study. Lancet Oncol. 2020;21:207-221.

59. Kumar S, Paiva B, Anderson KC, et al. International Myeloma Working Cohort consensus criteria for response and minimal residual disease assessment in multiple myeloma. Lancet Oncol. 2016;17: e328-e346.

60. Lonial S, Lee HC, Badros A, et al. DREAMM-2: single agent belantamab mafodotin in relapsed/refractory multiple myeloma refractory to proteasome inhibitors, immunomodulatory agents, and refractory and/or intolerant to anti-CD38 mAbs. HemaSphere. 2020;4 (S1):abstract EP970.

61. Tzogani K, Penttila K, Lahteenvuo J, et al. EMA review of belantamab mafodotin (Blenrep) for the treatment of adult patients with relapsed/refractory multiple myeloma. Oncologist. 2021;26:70-76.

62. Nooka A, Lee HC, Badros A, et al. Infusion-related reactions (IRRs) in the DREAMM-2 study of single-agent belantamab mafodotin (Belamaf) in patients with relapsed/refractory multiple myeloma (RRMM). Blood. 2020;136(Suppl. 1):abstract 3221.

63. Popat R, Lonial S, Voorhees PM, et al. DREAMM-2: single-agent belantamab mafodotin (Belamaf) effects on patient-reported outcome (PRO) measures in patients with relapsed/refractory multiple myeloma (RRMM). Blood. 2020;136(Suppl. 1):abstract2278.

64. Lee HC, Cohen AD, Chari A, et al. DREAMM-2: single-agent belantamab mafodotin (GSK2857918) in patients with relapsed/ refractory multiple myeloma (RRMM) and renal impairment. $J$ Clin Oncol. 2020;38(Suppl):abstract 8519.

65. Cohen AD, Trudel S, Lonial S, et al. DREAMM-2: single-agent belantamab mafodotin (GSK2857918) in patients with relapsed/ refractory multiple myeloma (RRMM) and high-risk cytogenetics. HemaSphere. 2020;4(S1):abstract EP1037.

66. Lonial S, Lee HC, Trudel S, et al. DREAMM-2: single-agent belantamab mafodotin in patients with relapsed/refractory multiple myeloma (RRMM)-1-year outcomes by prior therapies. Blood. 2020;136 (Suppl. 1):abstract 1417.

67. Cohen AD, Lee HC, Trudel S, et al. Impact of prolonged dose delays on response with belantamab mafodotin (Belamaf; GSK2857916) treatment in the in the DREAMM-2 study: 13-month follow-up. Clin Lymphoma Myeloma Leuk. 2020;20(Suppl. 1):abstractMM250.

68. Markham A. Belantamab Mafodotin: first approval. Drugs. 2020;80:1607-1613.

69. Ferron-Brady G, Rathi C, Collins J, et al. Therapeutic dose selection for belantamab mafodotin, a BCMA-targeting agent, in patients with relapsed/refractory multiple myeloma: application of population pharmacokinetics (PopPK) and exposure-response (E-R) analyses. Cancer Res. 2020;80(16 Suppl):abstract CT196.

70. Gandhi AK, Kang J, Havens CG, et al. Immunomodulatory agents lenalidomide and pomalidomide co-stimulate $\mathrm{T}$ cells by inducing degradation of $\mathrm{T}$ cell repressors Ikaros and Aiolos via modulation of the E3 ubiquitin ligase complex CRL4 ${ }^{\mathrm{CRBN}}$. Br J Haematol. 2014;164:811-821.

71. Trudel S, McCurdy A, Sutherland HJ, et al. Part 1 results of a dose finding study of belantamab mafodotin (GSK2857916) in combination with pomalidomide and dexamethasone for the treatment of relapsed/refractory multiple myeloma. Blood. 2020;136(Suppl. 1): abstract725. 
72. Richardson PG, Oriol A, Beksac M, et al. Pomalidomide, Bortezomib, and dexamethasone for patients with relapsed or refractory multiple myeloma previously treated with lenalidomide (OPTIMISMM): a randomised, open-label, phase 3 trial. Lancet Oncol. 2019;20:781-794.

73. Chen CI, Bahlis N, Gasparetto C, et al. Selinexor, pomalidomide and dexamethasone (SPd) in patients with relapsed or refractory multiple myeloma. Blood. 2019;134(Suppl. 1):abstract141.

74. Nooka AK, Mateos M-V, Bahlis N, et al. DREAMM-4: evaluating safety and clinical activity of belantamab mafodotin (belamaf) in combination with pembrolizumab in patients with relapsed/refractory multiple myeloma. HemaSphere. 2020;4(S1):abstract EP955.

75. Richardson PG, Nooka A, Quach H, et al. DREAMM-5 platform trial: belantamab mafodotin (belamaf) in combination with four different novel agents in patients with relapsed/refractory multiple myeloma (RRMM). Blood. 2020;136(Suppl. 1):abstract2999.

76. Popat R, Nooka A, Stockerl-Goldstein K, et al. DREAMM-6: safety, tolerability and clinical activity of bellantamab mafodotin (Belamaf) in combination with bortezomib/dexamethasone in relapsed/ refractory multiple myeloma (RRMM). Blood. 2020;136(Suppl. 1):abstract 1419.

77. Rifkin RM, Boyd K, Grosicki S, et al. DREAMM-7: a phase III study of the efficacy and safety of belantamab mafodotin (belamaf) with Bortezomib, and dexamethasone (B-Vd) in patients with relapsed/ refractory multiple myeloma (RRMM). Blood. 2020;136(Suppl. 1) abstract 3247.

78. Trudel S, Davis R, Lewis NM, et al. DREAMM-8: a phase III study of the efficacy and safety of belantamab mafodotin with pomalidomide and dexamethasone (B-Pd) vs pomalidomide plus Bortezomib and dexamethasone $(\mathrm{PVd})$ in patients with relapsed/refractory multiple myeloma (RRMM). Blood. 2020;136(Suppl. 1):abstract 2302.
79. Farooq AV, Degli Esposti S, Popat R, et al. Corneal epithelial findings in patients with multiple myeloma treated with antibody-drug conjugate belantamab mafodotin in the pivotal, randomized, DREAMM2 Study. Ophthalmol Ther. 2020;9:889-911.

80. Lonial S, Nooka A, Thulasi P, et al. Recovery of ocular events with longer-term follow-up in the DREAMMM-2 study of single-agent belantamab mafodotin (belamaf) in patients with relapsed or refractory multiple myeloma (RRMM). Blood. 2020;136(Suppl. 1):abstract 3224.

81. Becnel MR, Lee HC. The role of belantamab mafodotin for patients with relapsed and/or refractory multiple myeloma. Ther $A d v$ Hematol. 2020;11:1-11.

82. Ferron-Brady G, Rathi C, Collins J, et al. Exposure-response (E-R) for ocular safety endpoints for belantamab mafodotin (belamaf), a B-Cell Maturation Antigen (BCMA)-targeting agent, in patients with relapsed/refractory multiple myeloma (RRMM) in the DREAMM-2 study. Blood. 2020;136(Suppl. 1):abstract 1420.

83. Popat R, Warcel D, O’Nions J, et al. Characterization of response and corneal events with extended follow-up after belantamab mafodotin (GSK2857916) monotherapy for patients with relapsed multiple myeloma: a case series from the first-time-in-human clinical trial. Haematologica. 2020;105:e261-e263.

84. Lin H-P, Singla B, Ghoshal P, et al. Identification of novel micropinocytosis inhibitors using a rational screen of Food and Drug Administration-approved drugs. Br Journal of Pharmacology. 2018;175:3640-3655.

85. Dimopoulos MA, Moreau P, Terpos E, et al. Multiple myeloma: EHA-ESMO clinical practice guidelines for diagnosis, treatment and follow-up. Ann Oncol. 2021;32:309-322.
Drug Design, Development and Therapy

\section{Publish your work in this journal}

Drug Design, Development and Therapy is an international, peerreviewed open-access journal that spans the spectrum of drug design and development through to clinical applications. Clinical outcomes, patient safety, and programs for the development and effective, safe, and sustained use of medicines are a feature of the journal, which has also been accepted for indexing on PubMed Central. The manuscript management system is completely online and includes a very quick and fair peer-review system, which is all easy to use. Visit http://www. dovepress.com/testimonials.php to read real quotes from published authors. 